\title{
Comparing Raman mapping and electron microscopy for characterizing compositional gradients in thermoelectric materials
}

\author{
M. Yasseri* ${ }^{, 1,2,4}$, D. Schüpfer ${ }^{3,4}$, M. Weinhold ${ }^{3,4}$, L. Chen ${ }^{3,4}$, H. Kamila $^{2}$, E. Müller ${ }^{1,2,4}$, J. de \\ Boor $^{2}$, P. J. Klar*,3,4
}

${ }^{1}$ Institute of Inorganic and Analytical Chemistry, Justus Liebig University, Giessen, Germany
${ }^{2}$ German Aerospace Center (DLR), Institute of Materials Research, Cologne, Germany
${ }^{3}$ Institute of Experimental Physics I, Justus Liebig University, Giessen, Germany
${ }^{4}$ Center for Materials Research/LaMa, Justus Liebig University, Giessen, Germany
Corresponding Author(s): Mohammad Yasseri@dlr.de, Peter.J.Klar@exp1.physik.uni-giessen.de

We demonstrate that Raman mapping provides a cheap, fast, and non-invasive way of mapping compositional fluctuations occurring in state-of-the-art thermoelectric materials. Exemplarily, we discuss Raman results obtained on a $\mathrm{Mg}_{2} \mathrm{Si}-\mathrm{Mg}_{2} \mathrm{Sn}$ diffusion couple and compare resulting compositional mappings with those obtained on the same sample using electron microscopy in conjunction with energy-dispersive X-ray emission and back-scattered electrons as probes. We obtained the same level of quantitative information by the three approaches. The lateral resolution achieved in the Raman mappings can be as good as $1.4 \mu \mathrm{m}$ and typical recording times per data point are of the order of $50 \mathrm{~s}$.

Keywords: Raman spectroscopy; scanning electron microscopy; thermoelectric materials; magnesium tin silicide.

Many state-of-the-art thermoelectric materials are solid solutions or composite materials comprising several materials phases. Alloying, nanostructuring, and designing inclusions are major tools for enhancing the thermoelectric figure of merit $z T$ of such thermoelectric materials. In thermoelectric devices, the materials employed are typically exposed to large temperature 
gradients of the order of several hundred degrees Celsius per centimeter. Phase segregation and atomic interdiffusion are thus likely to occur in thermoelectric materials and means of characterizing these effects in situ and ex situ are essential for further optimization of the materials.

$\mathrm{Mg}_{2} \mathrm{Si}_{1-x} \mathrm{Sn}_{x}$ solid solutions belong to this class of state-of-the-art thermoelectric materials [1-4]. Their good thermoelectric performance make these compounds attractive candidates for thermoelectric generators (TEG) $[1,3]$. The highest figures of merit for $\mathrm{Mg}_{2} \mathrm{Si}_{1-x} \mathrm{Sn}_{x}$ with $z T=1.1$ to 1.5 have been reported for $x=0.6$ and 0.7 [1-5]. However, it is not clear whether the solid solutions of these compositions are thermodynamically stable at the application temperatures of $450^{\circ} \mathrm{C}$ to $600^{\circ} \mathrm{C}^{\circ} \mathrm{C}$.. There is a miscibility gap reported in the pseudo-binary phase diagram of the $\mathrm{Mg}_{2} \mathrm{Si}-\mathrm{Mg}_{2} \mathrm{Sn}$ material system. However, the results reported on the boundaries of the miscibility gap are disputed [6-8]. Recently, we carried out diffusion couple experiments to determine the boundaries of the miscibility gap of the $\mathrm{Mg}_{2} \mathrm{Si}-\mathrm{Mg}_{2} \mathrm{Sn}$ material system $[9,10]$ and found a miscibility gap of $\mathrm{Mg}_{2} \mathrm{Si}_{1-x} \mathrm{Sn}_{x}$ between $x=0.25 \pm 0.05$ and $0.65 \pm$ 0.05 at $600^{\circ} \mathrm{C}[11,12]$.

Spatially resolved phase identification is typically performed by a mapping system comprises a scanning electron microscope (SEM) and an energy dispersive X-ray (EDX) probe. Depending on excitation energy of the chemical elements and the accelerating voltage, EDX compositional mapping or line scanning with such mapping systems can be very time consuming. The same holds for mapping systems combining transmission electron microscopy (TEM) and EDX. These can provide excellent spatial resolution, but are even more restricted than SEM-EDX systems in the size of the area which can be analyzed in a reasonable space of time. Furthermore, both, SEM and TEM, are expensive techniques which require a high-vacuum (HV) or even an ultra-highvacuum (UHV) environment and often a time-consuming sample preparation [13]. In situ 
applicable, fast and cheap spatial mapping systems sensitive to chemical composition and segregated phases are highly desirable. Raman mapping may serve in many cases as an interesting alternative approach to electron-microscopy based mapping techniques.

Raman spectroscopy probes the dynamics of the atoms of molecules of solids whereas X-ray diffraction probes their static arrangement. Nevertheless, Raman spectroscopy is sensitive to the atomic arrangement as well as to chemical composition $[14,15]$ as the frequencies of the Raman bands contain important information about force constants between the atoms involved as well as their masses. As a consequence X-ray traces and Raman spectra contain a comparable amount of information about the material and both can yield compositional information about organic and inorganic materials [16]. Raman mapping is a widely employed technique [17-19].

The intensities and frequencies of the Raman modes of solid solutions often vary with composition and show clear trends in the Raman spectra $[14,20]$. In particular, the Raman-active modes of $\mathrm{Mg}_{2} \mathrm{Si}_{1-x} \mathrm{Sn}_{x}$ vary linearly as a function of composition $x$ [21]. On the basis of the knowledge of such a dependence Raman spectroscopy becomes a useful tool for spatial or in-situ mapping of compositional changes [14, 22-25]. Furthermore, Raman spectroscopy is noninvasive and nondestructive, and may be performed in atmosphere and in a wide temperature range without a specialized sample preparation [26]. [26].

In this study, we compare compositional mappings of the interface region of $\mathrm{Mg}_{2} \mathrm{Si}-\mathrm{Mg}_{2} \mathrm{Sn}$ diffusion couples obtained by Raman spectroscopy and SEM in conjunction with EDX and BSE probes. We demonstrate that Raman mapping is a viable alternative for assessing compositional gradients in $\mathrm{Mg}_{2} \mathrm{Si}_{1-x} \mathrm{Sn}_{x}$ thermoelectric materials fast and reliably.

Details of the synthesis of $\mathrm{Mg}_{2} \mathrm{Si}_{x} \mathrm{Sn}_{1-x}$ samples by mechanical alloying followed by direct current sintering have been given in our previous works [2, 12, 27, 28]. A diffusion couple was 
prepared by joining $\mathrm{Mg}_{2} \mathrm{Si}$ and $\mathrm{Mg}_{2} \mathrm{Sn}$ pellets at $600^{\circ} \mathrm{C}$ in the DSP for 10 hours. For a long term diffusion experiment, the diffusion couple was sealed in a quartz ampule under argon (560 mbar) and magnesium vapor pressure to prevent oxidation, contamination, and to suppress $\mathrm{Mg}$ loss [11, 29]. The sample was annealed at $600^{\circ} \mathrm{C}$ for approximately 110 hours. The microstructural and thermodynamic evolution of the phases formed in the diffusion couple was analyzed in detail [11].

Raman spectra of the diffusion couple were obtained at room temperature using a Renishaw inVia Raman microscope system equipped with a holographic notch filter for suppression of the Rayleigh scattered light and a single spectrometer for dispersing the Raman scattered light. A liquid nitrogen-cooled charge-coupled device detector was used for detection and either an Argon ion or a HeNe laser operating at either $514.5 \mathrm{~nm}$ or $632.8 \mathrm{~nm}$ were used for excitation. Raman spectra were taken with 10 seconds acquisition time and accumulated five times with a laser power of about $1 \mathrm{~mW}$ on the sample. Raman line scans were acquired from the $\mathrm{Mg}_{2} \mathrm{Si}-\mathrm{Mg}_{2} \mathrm{Sn}$ diffusion couple by scanning along the diffusion gradient, i.e., perpendicular to the interface over a length of up to100 $\mu \mathrm{m}$ and with typical step sizes of $0.1 \mu \mathrm{m}$ to $1 \mu \mathrm{m}$. The center of the line scan was located at the interface of the diffusion couple. In addition Raman mappings based on about 1200 Raman spectra obtained on rectangular areas of up to several tens $\mu \mathrm{m}^{2}$ were recorded (see Supplementary Material- Fig. S1). The data were analyzed quantitatively using the known compositional dependence of the $\mathrm{F}_{2 \mathrm{~g}}$ Raman mode $\omega\left(\mathrm{F}_{2 \mathrm{~g}}\right)=259 \mathrm{~cm}^{-1}-38 \mathrm{~cm}^{-1} \cdot x$ where $x$ is the composition of the bulk-like, unstrained $\mathrm{Mg}_{2} \mathrm{Si}_{1-x} \mathrm{Sn}_{x}$ solid solution.

Images or line scans of the sample surface were recorded in an electron microscope system using BSE as well as EDX as probes. The SEM system used was a Zeiss Ultra 55 SEM equipped with a Zeiss QBSE detector and an Oxford EDX detector (PentaFETx3). The spatial maps of the 
composition $x$ of $\mathrm{Mg}_{2} \mathrm{Si}_{x} \mathrm{Sn}_{1-x}$ were generated from the backscattered electron (BSE) image contrast as described previously [12].

An SEM image of the interface region of the $\mathrm{Mg}_{2} \mathrm{Si}-\mathrm{Mg}_{2} \mathrm{Sn}$ diffusion couple and a corresponding two-dimensional compositional map obtained using BSE as probe are plotted in the left graph of Fig. 1 [12]. The image height corresponds to a length of $100 \mu \mathrm{m}$ across the interface which basically divides the image into an upper and a lower half. An analysis, as reported in [11], yields that the main thermodynamic immiscibility range of $\mathrm{Mg}_{2} \mathrm{Si}_{1-x} \mathrm{Sn}_{x}$ (miscibility gap I) at $600^{\circ} \mathrm{C}$, lies between $0.25 \pm 0.05<x<0.65 \pm 0.05$ consistent with previous reports [30]. It manifests itself at the interface as an abrupt compositional change from $x=0.25 \pm 0.05$ to $x=0.65 \pm 0.05$. The compositional boundaries of the miscibility gap are given by the compositions of the phases coexisting at either side of the interface of the diffusion couple. The color change from red to yellow in the compositional map of Fig. 1 reflects this behavior. The color gradients on either side of the interface show that, going away from the interface, the solid solutions get either richer with Si (deeper red, going down) or richer with Sn (lighter yellow, going up), i.e., approach the compositions corresponding to the two binary starting materials $\mathrm{Mg}_{2} \mathrm{Si}$ (bottom of the image) and $\mathrm{Mg}_{2} \mathrm{Sn}$ (top of the image) of the diffusion couple. 


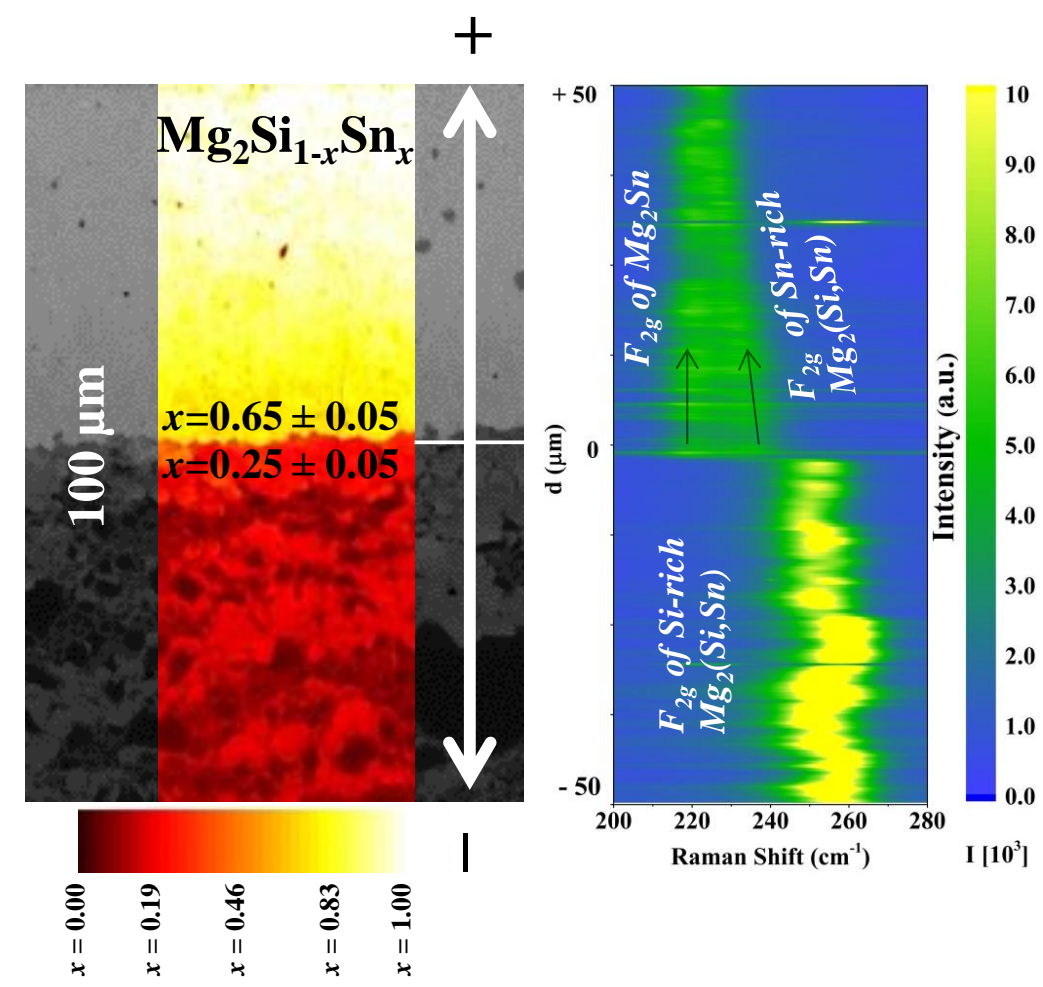

Fig. 1: Left: SEM image of the $\mathrm{Mg}_{2} \mathrm{Si}-\mathrm{Mg}_{2} \mathrm{Sn}$ diffusion couple and the corresponding two-dimensional color map of the composition extracted from the contrast of the back-scattered electrons. The composition step at the interface reflected by the abrupt color change is indicative of the miscibility gap between $x=0.25 \pm 0.05$ (red) and $x=0.65 \pm$ 0.05 (yellow). Right: Vertically stacked Raman spectra obtained by a line scan of a length of $100 \mu \mathrm{m}$ with a step size of $0.25 \mu \mathrm{m}$ across the diffusion zone, the colors indicate the Raman intensity. The vertical axis corresponds to the positions (distances from the interface) where the individual Raman spectra of the line scan were recorded. Distance $d=0 \mu \mathrm{m}$ corresponds to the position of the interface. The vertical axes in both images are on the same scale and directly comparable.

The color plot on the right of Fig. 1 shows the results of a Raman line scan over a length of 100 $\mu \mathrm{m}$ across the interfacial diffusion zone of the $\mathrm{Mg}_{2} \mathrm{Si}-\mathrm{Mg}_{2} \mathrm{Sn}$ diffusion couple. A step size of $0.25 \mu \mathrm{m}$ was used in the line scan. The spectral intensity of each spectrum is given in color coding along the horizontal wavenumber axis. 

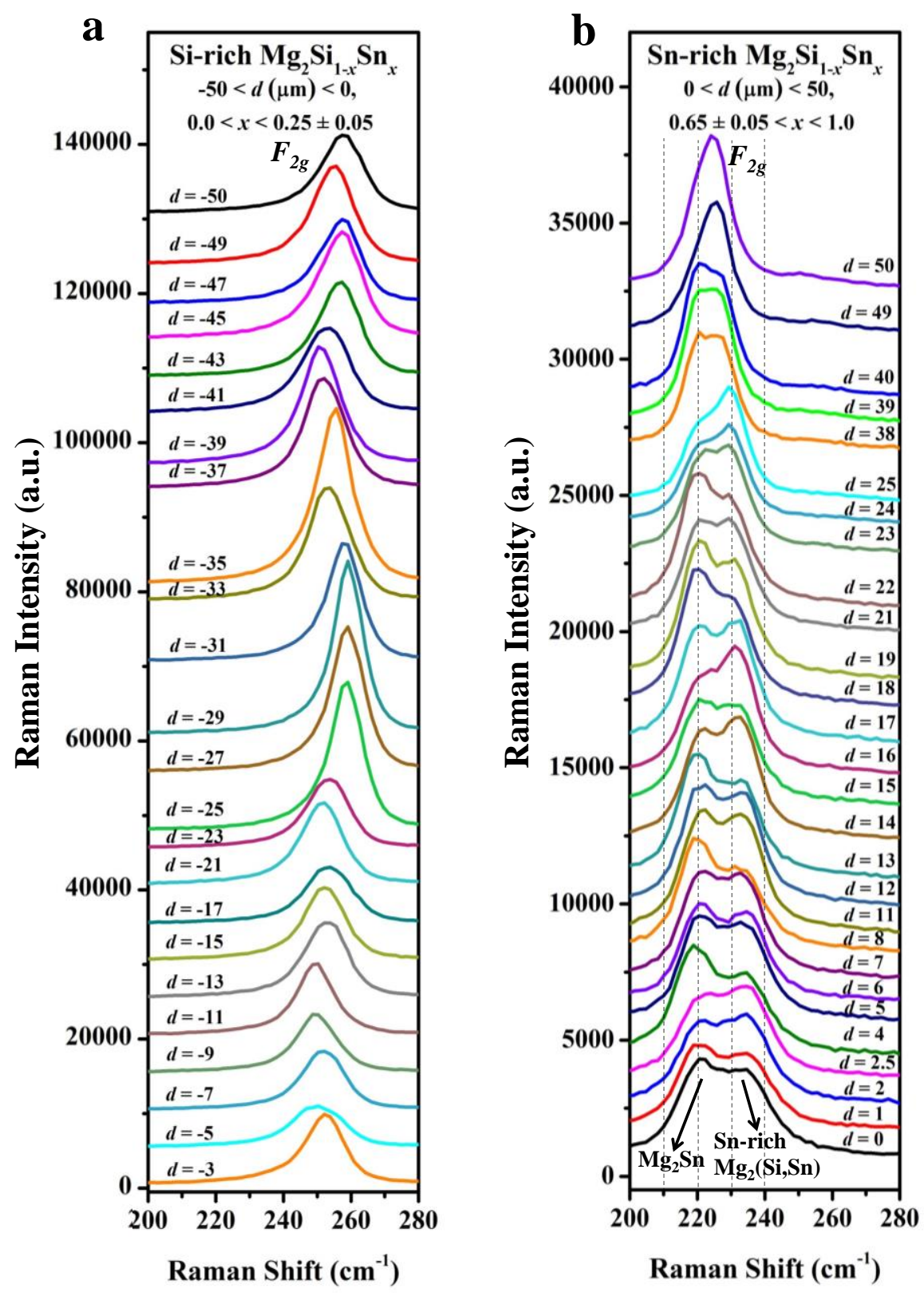

Fig. 2: Raman spectra of a line scan across the interface of a $\mathrm{Mg}_{2} \mathrm{Si}-\mathrm{Mg}_{2} \mathrm{Sn}$ diffusion couple located at $d=0 \mu \mathrm{m}$. a) Spectra of $\mathrm{Mg}_{2} \mathrm{Si}_{1-x} \mathrm{Sn}_{x}$ on the Si-rich side of the diffusion couple $(-50 \mu \mathrm{m}<d<0 \mu \mathrm{m})$. b) Spectra of $\mathrm{Mg}_{2} \mathrm{Si}_{1-x} \mathrm{Sn}_{x}$ on the Sn-rich side of the diffusion couple $(0 \mu \mathrm{m}<d<50 \mu \mathrm{m})$. The $\mathrm{F}_{2 \mathrm{~g}}$ mode of the Sn-rich solid solution at the interface of the diffusion couple and the $\mathrm{F}_{2 \mathrm{~g}}$ mode of co-existing almost stoichiometric $\mathrm{Mg}_{2} \mathrm{Sn}$ (formed from a Mg-Sn melt) are indicated by black arrows for the spectrum corresponding to $d=0 \mu \mathrm{m}$ in Fig. $2 \mathrm{~b}$. The parallel vertical dashed lines in Fig. $2 \mathrm{~b}$ are plotted as a guide to the eye. 
Clear trends in the series of Raman spectra can be detected in the right graph of Fig. 1 and even more clearly in Fig. 2 which shows waterfall plots of the Raman spectra recorded at the different positions between $\mathrm{d}=-50 \mu \mathrm{m}$ and $50 \mu \mathrm{m}$. The Raman spectra of the Sn-rich side exhibit a peak broadening and two Raman peaks can be identified in the range from 220 to $240 \mathrm{~cm}^{-1}$. One of the two peaks shifts from $240 \mathrm{~cm}^{-1}$ close to the interface to lower wavenumbers down to almost 220 $\mathrm{cm}^{-1}$ with increasing distance from the interface, whereas the other peak keeps its position at about $220 \mathrm{~cm}^{-1}$ in all Raman spectra for $d>0 \mu \mathrm{m}$. This constant Raman frequency basically corresponds to that expected for binary $\mathrm{Mg}_{2} \mathrm{Sn}$. The Raman-active $\mathrm{F}_{2 \mathrm{~g}}$ mode of $\mathrm{Mg}_{2} \mathrm{Si}_{1-\mathrm{x}} \mathrm{Sn}_{x}$ shows a one-mode behavior and varies continuously with $x$ keeping its extended character in the alloy [21]. This leads to a linear shift of the corresponding Raman mode frequency. Therefore, the presence of two peaks in the Raman spectra taken in the Sn-rich region of the diffusion couple indicates the co-existence of two phases of different composition in this part of the sample. We deduce that one of the phases corresponds to the Sn-rich matrix, where diffusion of $\mathrm{Si}$ occurred. The wavenumber corresponding to this phase shifts with the variation the composition along the line perpendicular to the interface, where a gradient in the composition exists. The peak corresponding to $\mathrm{Mg}_{2} \mathrm{Sn}$ arises from a second phase originating from a $\mathrm{Mg}-\mathrm{Sn}$ melt arising at the sintering temperature due to excess $\mathrm{Mg}$ on the $\mathrm{Mg}$-rich side of $\mathrm{Mg}_{2} \mathrm{Sn}$ which was added to compensate for $\mathrm{Mg}$ loss [11]. This secondary $\mathrm{Mg}_{2} \mathrm{Sn}$ phase was observed in form of solidified sub- $\mu \mathrm{m}$ droplets randomly distributed within the matrix on the Sn-rich side of the diffusion couple. A corresponding magnified SEM image of the Sn-rich side is shown in the supplementary material (Fig. S3).

The $\mathrm{Mg}_{2} \mathrm{Sn}$ droplets possess sizes below $400 \mathrm{~nm}$ and are randomly distributed in the sample. The laser spot is of a diameter of $1.4 \mu \mathrm{m}$ (see Fig. S4a) and, thus, averages over both phases, $\mathrm{Mg}_{2} \mathrm{Sn}$ and $\mathrm{Sn}$-rich $\mathrm{Mg}_{2}(\mathrm{Si}, \mathrm{Sn})$, yielding two peaks in the corresponding Raman spectrum. The intensity 
ratio of the two peaks in the Raman spectra shown in Fig. $4 \mathrm{~b}$ may vary with position as the number of $\mathrm{Mg}_{2} \mathrm{Sn}$ droplets may vary from one scan position to another.

In contrast, all Raman spectra taken at positions on the Si-rich side of the interface of the diffusion couple $(d<0 \mu \mathrm{m})$ exhibit a single peak corresponding to the $\mathrm{F}_{2 \mathrm{~g}}$ mode of a single phase exhibiting Raman shifts in the range between 245 and $260 \mathrm{~cm}^{-1}$. No significant broadening of the single Raman peaks is observed in the Si-rich region, but its peak position, i.e., frequency, oscillates as a function of distance from the interface. This behavior implies compositional fluctuations and structural inhomogeneity on top of a compositional gradient, which leads to a general shift towards higher wavenumbers with increasing distance from the interface approaching the $\mathrm{F}_{2 \mathrm{~g}}$ phonon frequency of binary $\mathrm{Mg}_{2} \mathrm{Si}$. The fluctuations in the Raman frequency on the Si-rich side of the diffusion couple likely are related to the presence of a second solubility gap (miscibility gap- II) for $\mathrm{Mg}_{2} \mathrm{Si}_{1-x} \mathrm{Sn}_{x}$. The higher and lower frequency limit correlate with the compositional boundaries of this miscibility gap which occurs between $0.05 \pm 0.05<x<0.15 \pm$ $0.05[11]$.

Raman spectra taken in the vicinity of the diffusion interface between $-1.0 \mu \mathrm{m}<d<1.0 \mu \mathrm{m}$ often exhibit three Raman bands (see Fig. S4b), two peaks corresponding to the $\mathrm{F}_{2 \mathrm{~g}}$ mode of Snrich $\mathrm{Mg}_{2} \mathrm{Si}_{1-x} \mathrm{Sn}_{x}$ and $\mathrm{Mg}_{2} \mathrm{Sn}$ on the Sn-rich side, and the third peak corresponding to the $\mathrm{F}_{2 \mathrm{~g}}$ mode of the Si-rich $\mathrm{Mg}_{2} \mathrm{Si}_{1-x} \mathrm{Sn}_{x}$ phase. This implies that in cases where all three peaks occur in a spectrum all three phases are present within the spot probed by the laser. Assuming an ideal interface, this implies that the spatial resolution is at least of the order of $1 \mu \mathrm{m}$ which is in accordance with the value of $1.4 \mu \mathrm{m}$ determined in Fig. S4a. 
The Sn content $x$ at each position of a Raman line scan or mapping can be extracted making use of the linear dependence of the Raman shift of the $\mathrm{F}_{2 \mathrm{~g}}$ mode in unstrained bulk $\mathrm{Mg}_{2} \mathrm{Si}_{1-x} \mathrm{Sn}_{x}$ solid solutions with $x$, i.e., $\omega\left(\mathrm{F}_{2 \mathrm{~g}}\right)=259 \mathrm{~cm}^{-1}-38 \mathrm{~cm}^{-1} \cdot x$ [21] (see Fig. S2). It should be noted that by using this dependence for $\omega\left(\mathrm{F}_{2 \mathrm{~g}}\right)$, we neglect effects due to stress which may arise at the interface of the diffusion couple. For example, hydrostatic pressure will lead to a blue shift of the Raman frequency [31] and uniaxial stress may lead to a mode splitting. Fig. 3 shows the extracted compositions of $\mathrm{Mg}_{2} \mathrm{Si}_{1-x} \mathrm{Sn}_{x}$ at each point of the line scan acquired across the diffusion zone in the range $-50 \mu \mathrm{m}<d<50 \mu \mathrm{m}$ with a step size of $1 \mu \mathrm{m}$. Composition values from EDX and BSE line scans [12] of a length of $100 \mu \mathrm{m}$ in the range $-50 \mu \mathrm{m}<d<50 \mu \mathrm{m}$ are also plotted in the same figure. It should be noted that the Raman line scan was recorded on a different, but comparable line across the interface than the other two line scans done in the SEM. The miscibility gap is indicated by the hatched region in the figure. It covers the compositional range of $0.27<x<0.63$. No Raman shift corresponding to compositions in this range was observed. The comparison of compositions extracted from the Raman data with those determined from EDX and BSE data indicates that the results of all three analysis methods agree very well. The remaining differences are very likely due to the fact that the three line scans are not acquired at the very same positions. It should be noted that, in particular the compositions extracted from the Raman and BSE data on the Si-rich side of the diffusion couple show clearly the spatial oscillation of the composition with an amplitude $\Delta x=0.1-0.2$ between $0.05 \pm 0.05<x<0.15 \pm$ 0.05 where the lower and upper limits for $x$ correspond to the boundaries of the second miscibility gap (miscibility gap- II) [11]. The good agreement of the compositions extracted from the Raman data with those obtained from the EDX and BSE data together with the finding that no mode splitting is discernible in Fig. 2 confirms our assumption made above. The impact of strain 
effects on the outcome of the Raman analysis is indeed negligible in case of the diffusion couple studied.

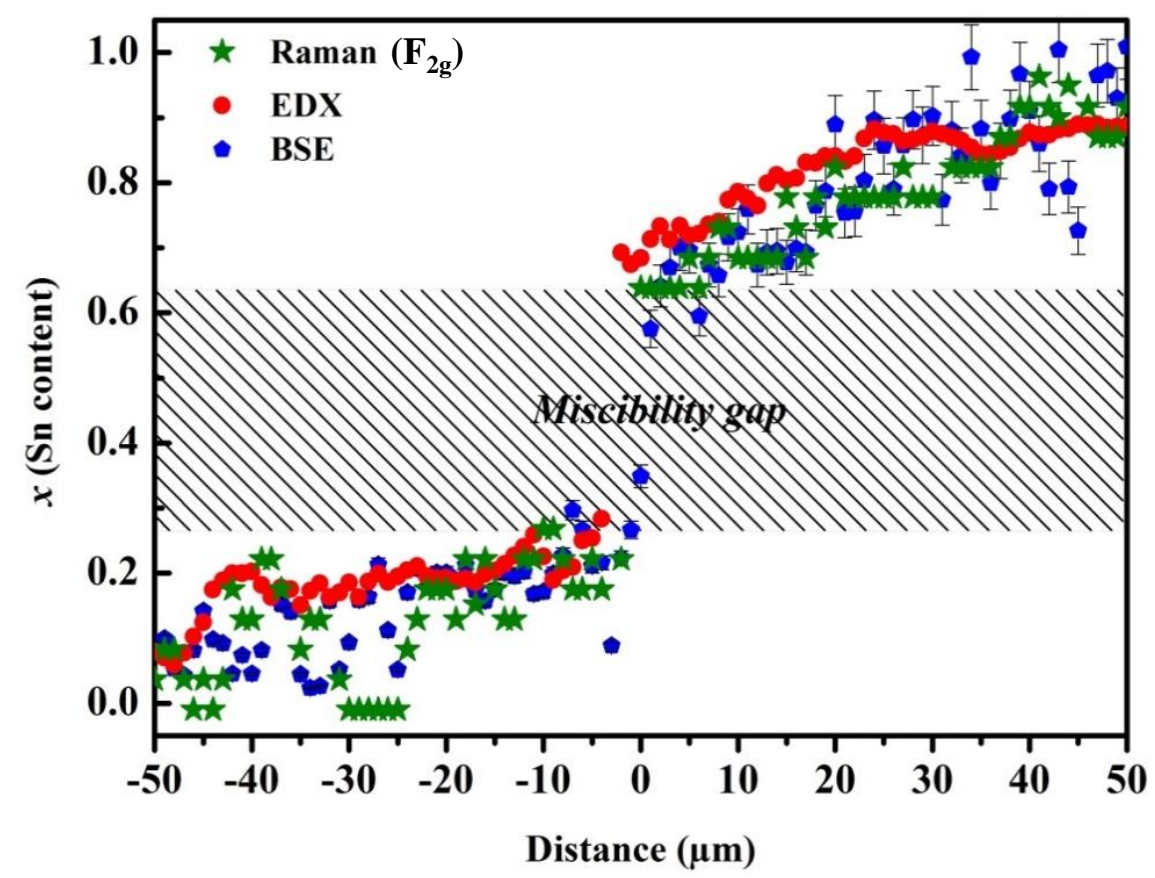

Fig. 3: Compositions along a line across the interface of a $\mathrm{Mg}_{2} \mathrm{Si}-\mathrm{Mg}_{2} \mathrm{Sn}$ diffusion couple, obtained by the analysis of data recorded by three different methods, i.e., Raman spectroscopy and scanning electron microscopy in combination with EDX or BSE as probes.

The Raman analysis was used as a tool for mapping the composition of $\mathrm{Mg}_{2} \mathrm{Si}_{1-x} \mathrm{Sn}_{x}$ across the diffusion zone of an $\mathrm{Mg}_{2} \mathrm{Si}-\mathrm{Mg}_{2} \mathrm{Sn}$ diffusion couple. The linear variation of the positions of the $\mathrm{F}_{2 \mathrm{~g}}$ Raman mode of $\mathrm{Mg}_{2} \mathrm{Si}_{1-x} \mathrm{Sn}_{x}$ with composition $x$ can be used for a fast and non-invasive determination of the composition profile across the interface of the diffusion couple. It shows the co-existence of two phases on the Sn-rich side of the diffusion couple, namely the Sn-rich $\mathrm{Mg}_{2} \mathrm{Si}_{1-x} \mathrm{Sn}_{x}$ solid solution matrix and almost stoichiometric sub- $\mu \mathrm{m} \mathrm{Mg} \mathrm{Mg}_{2} \mathrm{Sn}$ droplets (originating from a Mg-Sn melt formed during heat treatment). Furthermore, the Raman data confirm the miscibility gap between $0.27<x<0.63$. On the Si-rich side of the diffusion couple, the analysis 
reveals spatial oscillations of the composition due to a second miscibility gap between $0.05 \pm$ $0.05<x<0.15 \pm 0.05$. All these findings are in quantitative agreement with the analysis of data obtained by electron microscopy using EDX and BSE as probes. Thus, such a Raman analysis is an interesting alternative to electron microscopy techniques as the experiments may be performed under ambient conditions or potentially even in situ. This is not only of great interest for studies of degradation mechanisms of thermoelectric devices based on $\mathrm{Mg}_{2} \mathrm{Si}_{1-x} \mathrm{Sn}_{x}$ solid solutions, but also may be used for studying various other solid solutions employed as thermoelectric materials as long as they exhibit a characteristic compositional dependence of their Raman spectra.

\section{Acknowledgements}

Financial support is provided by the DFG via the GRK (Research Training Group) 2204 "Substitute Materials for sustainable Energy Technologies". The authors from DLR would like to acknowledge the endorsement from the DLR Executive Board Member for Space Research and Technology and the financial support from the Young Research Group Leader Program. Financial support of H. K. is provided by the DAAD.

\section{References}

[1] N. Farahi, S. Prabhudev, G.A. Botton, J.R. Salvador, H. Kleinke, ACS Appl Mater Interfaces 8(50) (2016) 34431-34437.

[2] A. Sankhla, A. Patil, H. Kamila, M. Yasseri, N. Farahi, E. Mueller, J. de Boor, ACS Applied Energy Materials 1(2) (2018) 531-542.

[3] V.K. Zaitsev, M.I. Fedorov, E.A. Gurieva, I.S. Eremin, P.P. Konstantinov, A.Y. Samunin, M.V. Vedernikov, Physical Review B 74(4) (2006).

[4] J. de Boor, T. Dasgupta, U. Saparamadu, E. Müller, Z.F. Ren, Materials Today Energy 4 (2017) 105-121.

[5] W. Liu, X. Tan, K. Yin, H. Liu, X. Tang, J. Shi, Q. Zhang, C. Uher, Phys Rev Lett 108(16) (2012) 166601.

[6] A. Kozlov, J. Gröbner, R. Schmid-Fetzer, Journal of Alloys and Compounds 509(7) (2011) 3326-3337.

[7] R. Viennois, C. Colinet, P. Jund, J.-C. Tédenac, Intermetallics 31 (2012) 145-151.

[8] I.-H. Jung, D.-H. Kang, W.-J. Park, N.J. Kim, S. Ahn, Calphad 31(2) (2007) 192-200.

[9] A.A. Kodentsov, G.F. Bastin, F.J.J. van Loo, J. Alloys Compd. 320(2) (2001) 207-217.

[10] J.S. Kirkaldy, Can. J. Phys. 36(7) (1958) 917-925.

[11] M. Yasseri, A. Sankhla, H. Kamila, R. Orenstein, D.Y.N. Truong, N. Farahi, J. de Boor, E. Mueller, Acta Mater. 185 (2020) 80-88.

[12] M. Yasseri, N. Farahi, K. Kelm, E. Mueller, J. de Boor, Materialia 2 (2018) 98-103. 
[13] J.J. Métois, S. Nitsche, J.C. Heyraud, Ultramicroscopy 27(4) (1989) 349-357.

[14] I.F. Chang, S.S. Mitra, Physical Review 172(3) (1968) 924-933.

[15] F.D. Hardcastle, I.E. Wachs, Journal of Raman Spectroscopy 21(10) (1990) 683-691.

[16] W.H. Weber, R. Merlin, Raman scattering in materials science, Springer Science \& Business Media2013.

[17] M. Nazari, M.W. Holtz, Applied Physics Reviews 5(4) (2018) 041303.

[18] I.-M. Chou, A. Wang, Journal of Asian Earth Sciences 145 (2017) 309-333.

[19] R.R. Jones, D.C. Hooper, L. Zhang, D. Wolverson, V.K. Valev, Nanoscale research letters 14(1) (2019) 231.

[20] R. da Costa, A. Rodrigues, P. Pizani, J. Alloys Compd. 697 (2017) 68-71.

[21] M. Yasseri, D. Schüpfer, L. Chen, H. Kamila, E. Mueller, J. de Boor, P.J. Klar, physica status solidi (RRL) - Rapid Research Letters (2019).

[22] A. Barker, Solid State Commun. 5 (1967) 695.

[23] F. Krüger, O. Reinkober, E. Koch-Holm, Annalen der Physik 390(1) (1928) 110-128.

[24] D. Feldman, M. Ashkin, J.H. Parker Jr, Phys. Rev. Lett. 17(24) (1966) 1209.

[25] H. Verleur, A. Barker Jr, Physical Review 149(2) (1966) 715.

[26] X. Zhu, T. Xu, Q. Lin, Y. Duan, Applied Spectroscopy Reviews 49 (2014) 64-82.

[27] H. Kamila, P. Sahu, A. Sankhla, M. Yasseri, H.-N. Pham, T. Dasgupta, E. Mueller, J. de Boor, Journal of Materials Chemistry A 7(3) (2019) 1045-1054.

[28] H. Kamila, A. Sankhla, M. Yasseri, N.P. Hoang, N. Farahi, E. Mueller, J. de Boor, Materials Today: Proceedings 8 (2019) 546-555.

[29] D. Kato, K. Iwasaki, M. Yoshino, T. Yamada, T. Nagasaki, Journal of Solid State Chemistry 258 (2018) 93-98.

[30] S. Vivès, P. Bellanger, S. Gorsse, C. Wei, Q. Zhang, J.-C. Zhao, Chemistry of Materials 26(15) (2014) 4334-4337.

[31] N.V. Morozova, S.V. Ovsyannikov, I.V. Korobeinikov, A.E. Karkin, K.-i. Takarabe, Y. Mori, S. Nakamura, V.V. Shchennikov, J. Appl. Phys. 115(21) (2014) 213705. 